PROCEEDINGS OF THE

AMERICAN MATHEMATICAL SOCIETY

Volume 132, Number 3, Pages 903-909

S 0002-9939(03)07021-7

Article electronically published on August 28, 2003

\title{
BORSUK-ULAM TYPE THEOREMS FOR COMPACT LIE GROUP ACTIONS
}

\author{
YASUHIRO HARA AND NORIHIKO MINAMI \\ (Communicated by Paul Goerss)
}

\begin{abstract}
Borsuk-Ulam type theorems for arbitrary compact Lie group actions are proven. The transfer plays a major role in this approach.
\end{abstract}

We present Borsuk-Ulam type theorems for arbitrary compact Lie group actions. The essence of our approach is a generalization of the ideal-valued index of FadellHusseini [FH88] using transfer [Boa66], BG75], Dol76], [KP72], Rou71]. Once an appropriate concept (Definition 0.3) is established in terms of transfer, then the proofs of our main results (Theorem 0.5. Theorem 0.6, and Corollary [0.9) are straightforward and simple.

We start with some standard definitions of the transfer and Gysin homomorphism.

Definition 0.1. (i) (cf. BG75, LMS86]) When $G$ is a compact Lie group, any compact $G$-manifold $F$ admits a $G$-embedding $F \subset W$ in a finite-dimensional real $G$-representation $W$ equipped with an invariant $G$-metric, by a theorem of Mostow [Mos57]. Then the Pontryagin-Thom construction gives us a map $t: S^{W} \rightarrow F^{\nu}$, where $\nu$ is the $G$-equivariant normal bundle of $F$. However, we prefer to rewrite this in the equivariant stable homotopy category [LMS86] as $t: S^{0} \rightarrow F^{-\tau(F)}$, where $\tau(F)$ is the tangent $G$-equivariant bundle of $G$ satisfying $\tau(F) \oplus \nu \cong F \times W$. Then for any honest $G$-equivariant vector bundle $\xi$ on $F$, we have the composite

$$
t(\xi): S^{0} \stackrel{\gamma}{\rightarrow} F^{-\tau(F)} \rightarrow F^{-\tau(F) \oplus \xi},
$$

where the second map between Thom spectra is induced by the inclusion of $G$ equivariant virtual bundles $-\tau(F) \subset-\tau(F) \oplus \xi$.

Given a $G$-CW complex $X$, smashing $t(\xi)$ with the identity of the suspension spectrum $\Sigma^{-\infty} X_{+}$yields another morphism, which is also denoted by $t(\xi)$ :

$$
t(\xi): \Sigma^{-\infty} X_{+} \rightarrow F^{-\tau(F) \oplus \xi} \wedge \Sigma^{-\infty} X_{+} .
$$

Received by the editors March 22, 2002 and, in revised form, August 12, 2002 and October 25, 2002.

2000 Mathematics Subject Classification. Primary 58E40, 55R12, 55N20; Secondary 55R35.

Key words and phrases. Borsuk-Ulam type theorems, transfer, generalized cohomology theories.

The second author was partially supported by Grant-in-Aid for Scientific Research No. 13440020, Japan Society for the Promotion of Science. 
(ii) We now specialize to the case $F=G / H$ and recall (e.g. [Kaw91, Th. 3.15]) that $\tau(G / H)$, the tangent $G$-bundle of $G / H$, is nothing but the bundle

$$
G \times_{H} \tau_{e H}(G / H) \rightarrow G / H
$$

associated with the principal $H$-bundle $G \rightarrow G / H$, where $\tau_{e H}(G / H)$, the tangent space of $G / H$ at $e H$, is regarded as the isotropy representation of $H$, and that an honest $G$-equivariant vector bundle on $G / H$ is nothing but a finite-dimensional real $H$-representation. Thus, for any finite-dimensional real representation $V$ of $H, t(V)$ may be written as

$t_{H}(V): \Sigma^{\infty} X_{+} \rightarrow(G / H)^{-\tau(G / H) \oplus V} \wedge \Sigma^{\infty} X_{+} \cong G_{+} \wedge_{H}\left(S^{-\tau_{e H}(G / H) \oplus V} \wedge \Sigma^{\infty} X_{+}\right)$, which is natural with respect to $X$ and is denoted by $t_{H}(V)$ to emphasize $H$.

Remark 0.2. (i) When $\xi=0, t(\xi)$ is the equivariant version of Boardman's umkehr map [Boa66], BG75, §4], which yields the equivariant Gysin type homomorphism in Definition 0.3 (ii). (For related constructions, consult [Kaw91, 6.1] for the equivariant Gysin homomorphism, and [ASi71, 3] for the family version of the topological index.) When $\xi=\tau(F), t(\xi)$ is the equivariant version of the Becker-Gottlieb transfer BG75].

(ii) When $G$ is a finite group and $F=G / H$, we will consider the case $\xi=0$, and the resulting transfer $t_{H}(\xi)$ is the equivariant version of the usual transfer of Roush [Rou71, Kahn-Priddy [KP72], and Becker-Gottlieb [BG75], which all agree in this case.

Now the following concept is the essence of our approach.

Definition 0.3. (i) Let $\tilde{h}_{G}$ be a $G$-equivariant reduced generalized cohomology theory. For any $G$-CW complex $X$, the pointed version $p_{X}: X_{+} \rightarrow S^{0}$ of the canonical projection $X \rightarrow\{$ a singleton $\}$ induces

$$
p_{X}^{*} ; \tilde{h}_{G}^{*}\left(S^{0}\right) \rightarrow \tilde{h}_{G}\left(X_{+}\right),
$$

and we call an element $x \in \tilde{h}_{G}^{*}\left(S^{0}\right)$ essential in $X$ if $p_{X}^{*}(x) \neq 0 \in \tilde{h}_{G}\left(X_{+}\right)$.

Following [FH88, Def. 2.1], we set the index of $X$ by

$$
\operatorname{Index}_{\tilde{h}_{G}^{*}}(X)=\operatorname{Ker}\left(p_{X}^{*} ; \tilde{h}_{G}^{*}\left(S^{0}\right) \rightarrow \tilde{h}_{G}\left(X_{+}\right)\right) .
$$

(ii) Let $\mathcal{F}$ be a family of conjugacy classes of proper closed subgroups of $G$, and $\tilde{\mathcal{F}}=\left\{\left((H), V_{H}\right) \mid(H) \in \mathcal{F}, V_{H}\right.$ : a finite-dimensional real representation of $\left.H\right\}$.

For a $G$-CW complex $X$, we say that an element $x \in \tilde{h}_{G}^{*}\left(S^{0}\right)$ is transferred from $\tilde{\mathcal{F}}$ in $X$ if $p_{X}^{*}(x) \in \tilde{h}_{G}^{*}\left(X_{+}\right)$is contained in the image of

$$
\begin{aligned}
\bigoplus_{\left((H), V_{H}\right) \in \tilde{\mathcal{F}}} t_{H}\left(V_{H}\right)^{*}: & \bigoplus_{\left((H), V_{H}\right) \in \tilde{\mathcal{F}}} \tilde{h}_{H}^{*}\left(S^{-\tau_{e H}(G / H) \oplus V_{H}} \wedge \Sigma^{\infty} X_{+}\right) \\
\cong & \bigoplus_{\left((H), V_{H}\right) \in \tilde{\mathcal{F}}} \tilde{h}_{G}^{*}\left(G_{+} \wedge{ }_{H}\left(S^{-\tau_{e H}(G / H) \oplus V_{H}} \wedge \Sigma^{\infty} X_{+}\right)\right) \\
\rightarrow & \tilde{h}_{G}^{*}\left(X_{+}\right) .
\end{aligned}
$$

We set the transfer index of $X$ with respect to $\tilde{\mathcal{F}}$ by

$$
\operatorname{Index}_{\tilde{h}_{G}^{*}}(X ; \tilde{\mathcal{F}})=\left(p_{X}^{*}\right)^{-1}\left(\operatorname{Im}\left(\bigoplus_{\left((H), V_{H}\right) \in \tilde{\mathcal{F}}} t_{H}\left(V_{H}\right)^{*}\right)\right) \subseteq \tilde{h}_{G}^{*}\left(S^{0}\right)
$$


which generalizes $\operatorname{Index}_{\tilde{h}_{G}^{*}}(X)$ in (i), for $\operatorname{Index}_{\tilde{h}_{G}^{*}}(X ; \emptyset)=\operatorname{Index}_{\tilde{h}_{G}^{*}}(X)$.

(iii) We call an element $x \in \tilde{h}_{G}^{*}\left(S^{0}\right) \tilde{\mathcal{F}}$-essential in $X$ if $x$ is not contained in the submodule generated by $\operatorname{Index}_{\tilde{h}_{G}^{*}}(X)$ and $\operatorname{Index}_{\tilde{h}_{G}^{*}}(\{$ a singleton $\} ; \tilde{\mathcal{F}})$.

When $\tilde{h}_{G}^{*}$ is a multiplicative theory, $\operatorname{Im}\left(\bigoplus_{\left((H), V_{H}\right) \in \tilde{\mathcal{F}}} t_{H}\left(V_{H}\right)^{*}\right)$ becomes an (not necessarily proper; cf. Remark 0.4 (ii)) ideal of $\tilde{h}_{G}^{*}\left(X_{+}\right)$by the projection formula [BG75, §5], and so $\operatorname{Index}_{\tilde{h}_{G}^{*}}(X ; \tilde{\mathcal{F}})$ is an (not necessarily proper) ideal of $\tilde{h}_{G}^{*}$.

Remark 0.4. (i) Examples of $\tilde{h}_{G}^{*}$ include the $R O(G)$-graded equivariant generalized cohomology theories [LMS86] and the Borel cohomology type theory $\tilde{h}^{*}\left(E G_{+} \wedge_{G}-\right)$ associated with a non-equivariant reduced generalized cohomology theory $\tilde{h}^{*}$. We denote the corresponding unreduced theories by $h_{G}^{*}$ and $h^{*}$ so that $\tilde{h}_{G}^{*}\left(X_{+}\right)=$ $h_{G}^{*}(X)$ and $\tilde{h}^{*}\left(Y_{+}\right)=h^{*}(Y)$, respectively.

(ii) When $G=U(n), \tilde{\mathcal{F}}=\left\{\left(T^{n}, 0\right)\right\}$, and $h_{G}^{*}=K_{G}^{*}$ (the equivariant $K$-theory), the equivariant Gysin homomorphism $t_{T^{n}}(0)^{*}$ yields, via the equivariant Thom isomorphism, the split surjection

$$
K_{T^{n}}^{*}(X) \rightarrow K_{U(n)}^{*}(X)
$$

for any finite $G$-CW complex $X$. This fact played a major role in ASe69.

We have now arrived at the main results of this paper.

Theorem 0.5. (i) Given $G$-CW complexes $X$ and $Y$, suppose there exists an element $x \in \tilde{h}_{G}^{*}\left(S^{0}\right)$ such that for some $\tilde{\mathcal{F}}$ as in Definition 0.3(ii),

(1) $x$ is not transferred from $\tilde{\mathcal{F}}$ in $X$,

(2) $x$ is transferred from $\tilde{\mathcal{F}}$ in $Y$.

Then there is no G-equivariant map from $X$ to $Y$.

(ii) Given $G$-CW complexes $X$ and $Y$, suppose there exists an element $x \in \tilde{h}_{G}^{*}\left(S^{0}\right)$ such that for some $\tilde{\mathcal{F}}$ as in Definition 0.3 (ii), (iii),

(1) $x$ is $\tilde{\mathcal{F}}$-essential in $X$,

(2) $x$ is transferred from $\tilde{\mathcal{F}}$ in $Y$.

Suppose furthermore that all the members of the corresponding family $\mathcal{F}$ are subconjugate to some proper closed subgroup $K$ of $G$. Then any $G$-equivariant map $f: X \rightarrow Y$, when regarded as a $K$-equivariant map by restriction, is not $K$ equivariantly null-homotopic even in the $K$-equivariant stable homotopy category.

Proof. (i) follows immediately from the naturality of $\bigoplus_{\left((H), V_{H}\right) \in \tilde{\mathcal{F}}} t_{H}\left(V_{H}\right)^{*}$ with respect to $X$.

For (ii), since $x \in \tilde{h}_{G}^{*}\left(S^{0}\right)$ is transferred from $\tilde{\mathcal{F}}$ in $Y$, there is some $y \in$ $\bigoplus_{\left((H), V_{H}\right) \in \tilde{\mathcal{F}}} \tilde{h}_{H}^{*}\left(S^{-\tau_{e H}(G / H) \oplus V_{H}} \wedge \Sigma^{\infty} Y_{+}\right)$such that

$$
\left(\bigoplus_{\left((H), V_{H}\right) \in \tilde{\mathcal{F}}} t_{H}\left(V_{H}\right)^{*}\right)(y)=p_{Y}^{*}(x) \in \tilde{h}_{G}^{*}\left(Y_{+}\right) .
$$

Without loss of generality, we may assume that $Y^{K} \neq \emptyset$ and choose a representative $H$ of $(H)$ to be a closed subgroup of $K$ for any $(H) \in \mathcal{F}$. Thus, consider a fixed $y_{0} \in Y^{K}\left(\subseteq Y^{H},(H) \in \mathcal{F}\right)$ as the basepoint, and we have

$$
\bigoplus_{\left((H), V_{H}\right) \in \tilde{\mathcal{F}}} \tilde{h}_{H}^{*}\left(S^{-\tau_{e H}(G / H) \oplus V_{H}} \wedge \Sigma^{\infty} Y_{+}\right)=\operatorname{Im} q_{Y}^{*} \oplus \operatorname{Im} p_{Y}^{*},
$$


where

$$
\begin{aligned}
q_{Y}^{*}: \tilde{h}_{H}^{*}\left(S^{-\tau_{e H}(G / H) \oplus V_{H}} \wedge \Sigma^{\infty} Y\right) \rightarrow \tilde{h}_{H}^{*}\left(S^{-\tau_{e H}(G / H) \oplus V_{H}} \wedge \Sigma^{\infty} Y_{+}\right), \\
p_{Y}^{*}: \tilde{h}_{H}^{*}\left(S^{-\tau_{e H}(G / H) \oplus V_{H}} \wedge \Sigma^{\infty} S^{0}\right) \rightarrow \tilde{h}_{H}^{*}\left(S^{-\tau_{e H}(G / H) \oplus V_{H}} \wedge \Sigma^{\infty} Y_{+}\right)
\end{aligned}
$$

are respectively induced by the $K$-equivariant pointed maps

$$
\begin{aligned}
& q_{Y}: Y_{+} \rightarrow Y, \\
& p_{Y}: Y_{+} \rightarrow S^{0},
\end{aligned}
$$

where $q_{Y}$ sends the extra basepoint of $Y_{+}$to $y_{0} \in Y$. In particular, for some $y^{\prime} \in$ $\tilde{h}_{H}^{*}\left(S^{-\tau_{e H}(G / H) \oplus V_{H}} \wedge \Sigma^{\infty} Y\right)$ and $y^{\prime \prime} \in \tilde{h}_{H}^{*}\left(S^{-\tau_{e H}(G / H) \oplus V_{H}} \wedge \Sigma^{\infty} S^{0}\right)$,

$$
y=q_{Y}^{*}\left(y^{\prime}\right)+p_{Y}^{*}\left(y^{\prime \prime}\right) \in \tilde{h}_{H}^{*}\left(S^{-\tau_{e H}(G / H) \oplus V_{H}} \wedge \Sigma^{\infty} Y_{+}\right)
$$

Now, contrary to the claim, suppose that

$$
q_{Y} \circ f_{+}: X_{+} \rightarrow Y
$$

is $K$-equivariantly stably null-homotopic. Then,

$$
\begin{aligned}
p_{X}^{*}(x)= & f_{+}^{*} \circ p_{Y}^{*}(x)=f_{+}^{*}\left(\left(\bigoplus_{\left((H), V_{H}\right) \in \tilde{\mathcal{F}}} t_{H}\left(V_{H}\right)^{*}\right)(y)\right) \\
= & \left(\bigoplus_{\left((H), V_{H}\right) \in \tilde{\mathcal{F}}} t_{H}\left(V_{H}\right)^{*}\right)\left(f_{+}^{*}(y)\right) \\
= & \left(\bigoplus_{\left((H), V_{H}\right) \in \tilde{\mathcal{F}}} t_{H}\left(V_{H}\right)^{*}\right)\left(f_{+}^{*} q_{Y}^{*}\left(y^{\prime}\right)+f_{+}^{*} p_{Y}^{*}\left(y^{\prime \prime}\right)\right) \\
= & \left(\bigoplus_{\left((H), V_{H}\right) \in \tilde{\mathcal{F}}} t_{H}\left(V_{H}\right)^{*}\right)\left(\left(q_{Y} \circ f_{+}\right)^{*}\left(y^{\prime}\right)+\left(p_{Y} \circ f_{+}\right)^{*}\left(y^{\prime \prime}\right)\right) \\
= & \left(\bigoplus_{\left((H), V_{H}\right) \in \tilde{\mathcal{F}}} t_{H}\left(V_{H}\right)^{*}\right)\left(0+\left(p_{X}\right)^{*}\left(y^{\prime \prime}\right)\right) \\
= & p_{X}^{*}\left(\left(\bigoplus_{\left((H), V_{H}\right) \in \tilde{\mathcal{F}}} t_{H}\left(V_{H}\right)^{*}\right)\left(y^{\prime \prime}\right)\right),
\end{aligned}
$$

which implies that

$$
x=\left(\bigoplus_{\left((H), V_{H}\right) \in \tilde{\mathcal{F}}} t_{H}\left(V_{H}\right)^{*}\right)\left(y^{\prime \prime}\right)+z
$$

for some $z \in \operatorname{Ker} p_{X}^{*}=\operatorname{Ind}_{\tilde{h}_{G}^{*}}(X)$. But, this means that $x$ is not $\tilde{\mathcal{F}}$-essential, which is a contradiction. This completes the proof.

Theorem 0.5 may be restated in terms of the index of Definition 0.3 .

Theorem 0.6. (i) (cf. [FH88, 2.2(a)]) Given $G$ - $C W$ complexes $X$ and $Y$, suppose that $\operatorname{Index}_{\tilde{h}_{G}^{*}}(Y ; \tilde{\mathcal{F}})$ is not contained in $\operatorname{Index}_{\tilde{h}_{G}^{*}}(X ; \tilde{\mathcal{F}})$ for some $\tilde{\mathcal{F}}$.

Then there is no $G$-equivariant map from $X$ to $Y$.

(ii) Given $G$-CW complexes $X$ and $Y$, suppose that $\operatorname{Index}_{\tilde{h}_{G}^{*}}(Y ; \tilde{\mathcal{F}})$ is not contained in the submodule generated by $\operatorname{Index}_{\tilde{h}_{G}^{*}}(X)$ and $\operatorname{Index}_{\tilde{h}_{G}^{*}}(\{$ a singleton $\} ; \tilde{\mathcal{F}})$ for some $\tilde{\mathcal{F}}$ such that all the members of the corresponding family $\mathcal{F}$ are sub-conjugate to some proper closed subgroup $K$ of $G$. Then any $G$-equivariant map $f: X \rightarrow Y$, when regarded as a $K$-equivariant map by restriction, is not $K$-equivariantly nullhomotopic even in the $K$-equivariant stable homotopy category. 
Example 0.7. (i) Let an elementary abelian 2-group $G=(\mathbb{Z} / 2)^{r}$ act freely on $S^{n_{1}-1} \times S^{n_{2}-1} \times \cdots \times S^{n_{r}-1}$ via the diagonal antipodal action, and consider the Borel cohomology $\tilde{H}_{(\mathbb{Z} / 2)^{r}}^{*}(-)=\tilde{H}^{*}\left(E(\mathbb{Z} / 2)_{+}^{r} \wedge_{(\mathbb{Z} / 2)^{r}}-; \mathbb{Z} / 2\right)$. Then,

$$
\begin{aligned}
\tilde{H}_{(\mathbb{Z} / 2)^{r}}^{*}\left(S^{0}\right) & =\tilde{H}^{*}\left(E(\mathbb{Z} / 2)_{+}^{r} \wedge_{(\mathbb{Z} / 2)^{r}} S^{0} ; \mathbb{Z} / 2\right)=H^{*}\left(B(\mathbb{Z} / 2)^{r} ; \mathbb{Z} / 2\right) \\
& =\mathbb{Z} / 2\left[x_{1}, x_{2}, \cdots, x_{r}\right]
\end{aligned}
$$

and

$$
\begin{aligned}
\operatorname{Index}_{\tilde{H}_{(\mathbb{Z} / 2)^{r}}^{*}}\left(S^{n_{1}-1} \times S^{n_{2}-1} \times \cdots \times S^{n_{r}-1}\right) & =\left(x_{1}^{n_{1}}, x_{2}^{n_{2}}, \cdots, x_{r}^{n_{r}}\right), \\
\operatorname{Index}_{\tilde{H}_{(Z / 2) r}^{*}}^{*}(\{\text { a singleton }\} ;\{(\{e\}, 0)\}) & =0, \\
\operatorname{Index}_{\tilde{H}_{(\mathbb{Z} / 2)^{r}}^{*}}\left(S^{n_{1}-1} \times S^{n_{2}-1} \times \cdots \times S^{n_{r}-1} ;\{(\{e\}, 0)\}\right) & =\left(x_{1}^{n_{1}}, x_{2}^{n_{2}}, \cdots,\right. \\
& \left.x_{r}^{n_{r}}, x_{1}^{n_{1}-1} x_{2}^{n_{2}-1} \cdots x_{r}^{n_{r}-1}\right) .
\end{aligned}
$$

Thus, if there is a $(\mathbb{Z} / 2)^{r}$-equivariant map

$$
S^{n_{1}-1} \times S^{n_{2}-1} \times \cdots \times S^{n_{r}-1} \rightarrow S^{m_{1}-1} \times S^{m_{2}-1} \times \cdots \times S^{m_{r}-1},
$$

where the target is also given by the diagonal antipodal action, then

$$
n_{1} \leq m_{1}, n_{2} \leq m_{2}, \cdots, n_{r} \leq m_{r}
$$

Furthermore, if all of these inequalities happen to be equalities, then any such $(\mathbb{Z} / 2)^{r}$-equivariant map (0.1) is non-equivariantly essential.

(ii) Let the $r$-dimensional torus $T^{r}$ act freely on $S^{2 n_{1}-1} \times S^{2 n_{2}-1} \times \cdots \times S^{2 n_{r}-1}$ via the diagonal standard action, and consider the Borel cohomology $\tilde{H}_{T^{r}}^{*}(-)=$ $\tilde{H}^{*}\left(E T_{+}^{r} \wedge_{T^{r}}-; \mathbb{Z}\right)$. Then a similar argument such as (i) implies that any $T^{r_{-}}$ equivariant self map of $S^{2 n_{1}-1} \times S^{2 n_{2}-1} \times \cdots \times S^{2 n_{r}-1}$ is a $T^{r}$-equivariant selfhomotopy equivalence.

We shall end this paper with an application that uses the Borel cohomology. For this purpose, we prepare a lemma.

Lemma 0.8. Let $\tilde{h}^{*}$ be a non-equivariant reduced generalized cohomology theory, and $X$ a $G-C W$ complex. Suppose there is an integer $n$ such that the canonical pointed projection $p_{X}: X_{+} \rightarrow S^{0}$ induces an isomorphism

$$
p_{X}^{*}: \tilde{h}^{d}\left(S^{0}\right) \rightarrow \tilde{h}^{d}\left(X_{+}\right)
$$

for any $d \leq n$. Then, for any

$$
\tilde{\mathcal{F}}=\left\{\left((H), V_{H}\right) \mid(H) \in \mathcal{F}, V_{H}: \text { a finite-dimensional real representation of } H\right\}
$$

with $V_{H}=\tau(G / H)$ for any $(H) \in \mathcal{F}$ (thus the Becker-Gottlieb situation as in Remark 0.2(i)), the canonical injection

$$
p_{X}^{*}: \operatorname{Index}_{\tilde{h}_{G}^{*}}(\{\text { a singleton }\} ; \tilde{\mathcal{F}}) \rightarrow \operatorname{Index}_{\tilde{h}_{G}^{*}}(X ; \tilde{\mathcal{F}})
$$

is an isomorphism in dimensions $\leq n$.

Proof. Considering the Atiyah-Hirzebruch spectral sequence

$$
E_{2}^{s, t}=H^{s}\left(B H ; h^{t}(X)\right) \Longrightarrow h^{s+t}\left(E H \times_{H} X\right),
$$

we see by the assumption that $p_{X}$ induces isomorphisms

$$
p_{X}^{*}: \tilde{h}^{d}\left(E H_{+} \wedge_{H} S^{0}\right) \rightarrow \tilde{h}^{d}\left(E H_{+} \wedge_{H} X_{+}\right)
$$


for any $d \leq n$. Now the claim follows immediately by applying the naturality of the map $\bigoplus_{\left((H), V_{H}\right) \in \tilde{\mathcal{F}}} t_{H}\left(V_{H}\right)^{*}$ with respect to $p_{X}: X_{+} \rightarrow S^{0}$.

From Lemma 0.8 and Theorem 0.5 we immediately get

Corollary 0.9. Let $X$ be a $G$-CW complex such that there is a non-equivariant reduced generalized cohomology theory $\tilde{h}^{*}$ and an integer $n$ so that the canonical pointed projection $p_{X}: X_{+} \rightarrow S^{0}$ induces an isomorphism

$$
p_{X}^{*}: \tilde{h}^{d}\left(S^{0}\right) \rightarrow \tilde{h}^{d}\left(X_{+}\right)
$$

for any $d \leq n$. Then, for any $G-C W$ complex $Y$, the following hold.

(i) Suppose there exists an element $x \in \tilde{h}_{G}^{n}\left(S^{0}\right)$ such that for some $\tilde{\mathcal{F}}$ with $V_{H}=$ $\tau(G / H)$ for any $(H) \in \mathcal{F}$ (thus the Becker-Gottlieb situation as in Remark 0.2(i))

(1) $x$ is not transferred from $\tilde{\mathcal{F}}$ in \{a singleton\},

(2) $x$ is transferred from $\tilde{\mathcal{F}}$ in $Y$.

Then there is no G-equivariant map from $X$ to $Y$.

(ii) Suppose there exists an element $x \in \tilde{h}_{G}^{n}\left(S^{0}\right)$ such that for some $\tilde{\mathcal{F}}$ with $V_{H}=$ $\tau(G / H)$ for any $(H) \in \mathcal{F}$ (thus the Becker-Gottlieb situation as in Remark 0.2(i))

(1) $x$ is $\tilde{\mathcal{F}}$-essential in $\{$ a singleton\},

(2) $x$ is transferred from $\tilde{\mathcal{F}}$ in $Y$.

Suppose furthermore that all the members of the corresponding family $\mathcal{F}$ are subconjugate to some proper closed subgroup $K$ of $G$. Then any $G$-equivariant map $f: X \rightarrow Y$, when regarded as a $K$-equivariant map by restriction, is not $K$ equivariantly null-homotopic even in the $K$-equivariant stable homotopy category.

\section{REFERENCES}

[ASe69] M. F. Atiyah and G. B. Segal, Equivariant K-theory and completion, J. Differential Geometry 3 (1969), 1-18. MR 41:4575

[ASi71] M. F. Atiyah and I. M. Singer, The index of elliptic operators: IV, Ann. of Math. 93 (1971), 119-138. MR 43:5554

[BG75] J. C. Becker and D. H. Gottlieb, The transfer map and fiber bundles, Topology 14 (1975), 1-12. MR 51:14042

[Boa66] J. M. Boardman, Stable Homotopy Theory (mimeographed), University of Warwick, 1966.

[Dol76] A. Dold, The fixed point transfer of fibre-preserving maps, Math. Z. 148 (1976), 215-244. MR 55:6416

[FH88] E. Fadell and S. Husseini, An ideal-valued cohomological index theory with applications to Borsuk-Ulam and Bourgin-Yang theorems, Ergodic Theory Dynam. Systems 8 (1988), 73-85. MR 89k:55002

[KP72] D. S. Kahn and S. B. Priddy, Applications of the transfer to stable homotopy theory, Bull. Amer. Math. Soc. 78 (1972), 981-987. MR 46:8220

[Kaw91] K. Kawakubo, The Theory of Transformation Groups, The Clarendon Press, Oxford University Press, 1991. MR 93g:57044

[Kob86] T. Kobayashi, The Borsuk-Ulam theorem for a $Z_{q}$-map from a $\mathbb{Z}_{q}$-space to $S^{2 n+1}$, Proc. Amer. Math. Soc. 97 (1986), 714-716. MR 87g:55001

[LMS86] L. G. Lewis, J. P. May, and M. Steinberger, Equivariant Stable Homotopy Theory, Lecture Notes in Math. No. 1213, Springer-Verlag, Berlin, Heidelberg, New York, 1986. MR 88e:55002 
[Mos57] G. D. Mostow, Equivariant embeddings in Euclidean space, Ann. Math. 65 (1957), 432446. MR 19:291c

[Rou71] F. W. Roush, Transfer in generalized cohomology theories, Ph.D. thesis, Princeton University, 1971.

Department of Mathematics, Graduate School of Science, Osaka University, Osaka, JAPAN

E-mail address: hara@math.sci.osaka-u.ac.jp

Department of Mathematics, Nagoya Institute of Technology, Gokiso, Showa-ku, NAGOYA 466-8555, JAPAN

E-mail address: minami.norihiko@nitech.ac.jp 